\title{
Mental Computation with Rational Numbers: Students' Mental Representations
}

\author{
Renata Carvalho \\ João Pedro da Ponte \\ Instituto de Educação, Universidade de Lisboa
}

We construct mental representations of the world that surround us, which we use to understand reality and make inferences. These mental representations reflect our mathematical and real world knowledge, and both are essential to perform mental computation and to relate numbers and operations. The aim of this study is to analyze students' mental representations underlying their mental computation strategies with rational numbers. Using a design research approach, we undertook two cycles of experimentation involving two teachers and 39 grade 6 students. The results suggest that students' mental computation strategies are mostly based on numerical relationships where descriptive representations play an important role. School contexts and daily life experience, where students' are involved when learning about numbers and operations, seem to be key promoters of mental representations.

Keywords: Mental computation, rational numbers, mental representations, student strategies.

Mental or internal representations are part of human cognitive structures (Johnson-Laird, 1990). Each individual stores in his/her mind a set of semantic contents (e.g., the meaning of some words, concepts about the world, etc.) and skills (e.g., drive a car, ride a bike, solving problems, etc.) that are activated by the working memory whenever we think (Plasencia, 2002). Through mental representations, we give meaning to phenomena and explain concepts and mathematical ideas. The knowledge we have about the world depends on mental representations that we create from learning experiences. Working memory, as a temporary system, depends on other systems including those involved in long-term memory. It is a mechanism of storing and processing information, which plays a fundamental role in cognitive tasks such as reasoning, learning, and understanding (Baddeley, 1993). The working memory is important for learning in general, but is even more important in mathematics learning, since mathematical reasoning is a high level cognitive activity that makes use of existing knowledge and basic facts stored in the long-term memory (Baddeley, 1993; Dehaene, 1997). 
Given the fundamental role of mental representations in our thinking, in learning rational numbers it is important to understand what mental representations students have in their mind when they compute mentally. The identification of students' mental representations may allow understanding what may be significant learning contexts (Prediger, 2008). Addressing this issue, we aim to analyze students' mental representations underlying their mental computation strategies with rational numbers. In a specific way, we want to analyze: what mental representations are students using in their mental computation strategies with rational numbers?

\section{Mental Representations}

In the perspective of Dehaene (1997), memory plays a central role in mental computation, not only for its ability to store numerical facts, but also for the mental models that it creates, based on previous knowledge and supporting students' reasoning. In the learning process, the context of tasks in which the numbers are presented to students also plays an important role. A structured mathematical knowledge, normally, is related to the context in which it is learned and students often have difficulties to apply this knowledge to new situations (Bell, 1993), especially when a new numerical set is learned. For Prediger (2008), these difficulties are related to an absence of mental models that can support students' reasoning. For example, students can use previous knowledge about whole numbers to work with rational numbers, but a discontinuity of mental models previously created in working with whole numbers, as Prediger (2008) indicates, requires a conceptual change approach. According to Prediger, students can link repeated addition to multiplication in the set of whole numbers, but an absence of a corresponding mental model for fraction multiplication requires a reconceptualization. This happens because in multiplying two fractions there is no repeated addition. Another example concerns fractions that can be interpreted as a "part-of" which also do not have a correspondence when working with whole numbers. For Prediger (2008), this is the basis of students' misconceptions and difficulties in learning rational numbers. Hence, to learn rational numbers, a conceptual change regarding whole numbers is needed that students can create new mental representations.

Johnson-Laird's (1990) theory of mental models attempts to explain complex knowledge processes, in particular, understanding and inference. This theory assumes that there are three types of mental representations according to their specificity and function: mental models, mental images, and propositional representations. If these mental models represent the real world with some specificity, they are considered images. If they make inferences about the real world represented by mental models, they are propositional representations. Mental models are mental representations created to make sense of personal experiences, which is the base of knowledge construction 
(Schnotz, Baadte, Müller, \& Rasch, 2010). Images are special sets of models that represent objects and correspond to a vision of the models as a result of perception or imagination. They represent the noticeable characteristics of real-world objects. Models as images are highly specific. For example, usually it is not possible to create a picture of a triangle without associating it to a particular triangle (equilateral, isosceles or scalene). Propositional representations refer to propositions that are used to make inferences. These propositions can be true or false and represent statements that do not correspond directly to the object represented (different structures), but they are important to establishing relationships.

For Plasencia (2002), internal and external representations are strongly related in working in mathematics. As we think, we make unconscious connections between our mental representations and our perceptions of external representations. As the author indicates, this relation allows us to analyze and interpret students' work. When a student uses an external representation to communicate, this is a partial reflection of what he/she has built in his/her mind (mental representation). As these representations are in the mind of each individual and are not directly observable, all we can say about them is based on inferences made from students' explanations (external representations).

Schnotz and Bannert (2003) classify external representations as descriptive or depictive. Descriptive representations are symbols, which have no relation to the objet that they represent, but enable us to understand relationships. Natural language, spoken or written, mathematical expressions or formulas are descriptive representations, which Schnotz et al. (2010) relate to propositional representations. Depictive representations are icons such as photographs, drawings, paintings, maps, or line graphs associated to the object that they represent by analogy or similarity, which Schnotz et al. (2010) relate to mental models and images. As we built mental (internal) representations largely from the interpretation of external representations and we use external representations to express our mental (internal) representations, Schnotz and Bannert (2003) consider that the distinction between descriptive and depictive made by them can be applied to mental representations. To clarify some possible confusion between these two notions, they present the following argument:

On the one hand, the mental representation of a text surface constructed during the processing of a spoken or written text and the propositional representation of the text contents are internal descriptions, since they describe the represented object with the help of symbols. On the other hand, visual images and mental models are internal depictions, since inherent structural characteristics are used here for the purpose of representation. (p. 143) 
This argument highlights the complementarity between mental (internal) and external representations, although both representations serve different purposes (Schnotz et al., 2010). While descriptive representations are more general, abstract, and powerful to express abstract knowledge, depictive representations are more concrete, specific, and selective. They are essential to achieve high levels of abstraction, creative thinking, understanding, arguing, reasoning, and problem solving skills. Sometimes, a depictive representation (model or image) allows us to create a simple descriptive representation (propositional representation) by facilitating a quick access to a symbolic process.

\section{Mental Computation Strategies with Rational Numbers}

To compute mentally, students need to understand the magnitude and value of numbers and the effect of operations on numbers, as well as to acquire a set of numerical facts that enable them to compute quickly and accurately (Heirdsfield, 2011). In this study, mental computation is seen as an exact computation made mentally in a quick and effective way, using mental representations and involving numerical facts, memorized rules, and relationships between numbers and operations. These ideas are reflected in our conceptual framework (Figure 1).

Numerical facts used by students in mental computation involve, for example, knowledge about the results of some operations (sums, differences, products, and quotients) or relationships among numbers and operations that they have stored in their memory throughout school learning experiences. Students' increasing knowledge and experience about numbers and operations, leads them to simplify computations more and more. This and the complexity of reasoning used in mental computation with rational numbers (Barnett-Clarke, Fisher, Marks, \& Ross, 2010), also leads them to use strategies based on memorized rules. Those rules are based on the application of procedures such as multiplying/dividing by powers of 10 (e.g., to calculate $10 \%$ of 350 , a student says, "It is 35 . I took out the zero"), or carrying out procedures with rational numbers (such as the rule of "invert-and-multiply" in the division of fractions).

Strategies based on numerical relationships reflect students' relational thinking (Empson, Levi, \& Carpenter, 2010), an important support for mental computation. Relational thinking refers to the ability to use fundamental properties of operations and the notion of equality to analyze and solve problems in a given context (Empson et al., 2010). Some numerical relationship strategies used by students are related to changing rational number representations (Caney \& Watson, 2003) (fraction $\rightarrow$ decimal; decimal $\rightarrow$ fraction; fraction $\rightarrow$ percent; percent $\rightarrow$ fraction; percent $\rightarrow$ decimal and decimal $\rightarrow$ percent) or a rational number to a whole number (decimal $\rightarrow$ whole number concerning 10/100). Students also use strategies 
such as part-whole, part-part relationship, equivalence between number sentences and the inverse relationship between operations. Numerical facts and memorized rules can arise as mental computation strategies, per se, but they also arise as an important support in establishing relationships between numbers and operations and vice-versa.

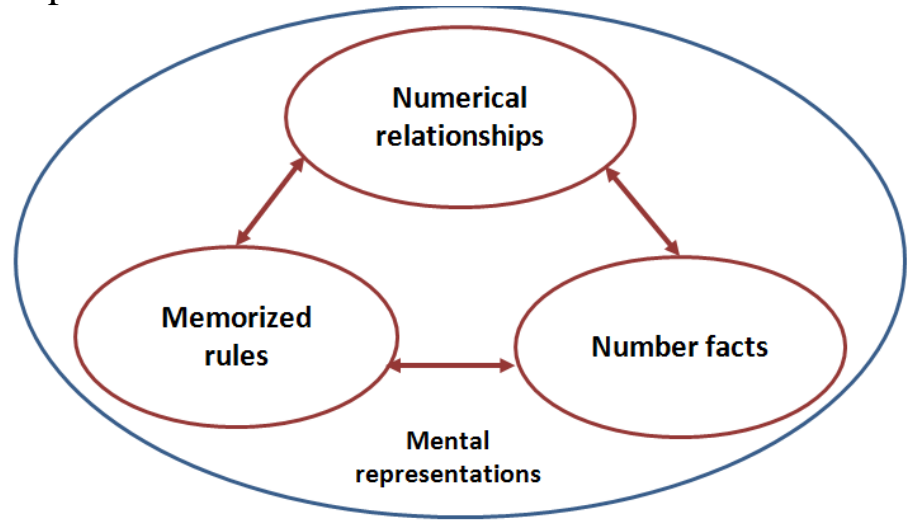

Figure 1. Conceptual framework.

Another important idea to consider in mental computation is the fact that we construct mental representations from the world that surrounds us, which we use in sense making and in making inferences as we highlighted in the previous section. Identifying students' mental representations may help us to understand their relational thinking, because they use mental representations to relate numbers and operations with their real world knowledge, including knowledge about mathematics. The mental representations that underlie students' strategies include mental models, mental images, and propositional representations (Johnson-Laird, 1990). Mental models are general perceptions of the world (e.g., using a context of sales to compute $20 \%$ of 25 ). Images involve a more specific perception of a real world object, in which some characteristics are considered (e.g., relating the symbolic representations $1 / 2$ to a pizza divided in two parts, taking only one part), and propositional representations represent true/false propositions that play an important role in the inference process (e.g., compute $40 \%$ of ? = 48 using a sequence of propositions leading to the solution: 'if 48 are $40 \%, 48$ $\div 4$ are $10 \%$ that is 12 . So, $10 \% \times 10$ is $100 \%$ and $12 \times 10$ is 120 the missing value').

\section{Methodology}

This study is developmental, qualitative, and interpretative, with a design research approach (Cobb, Confrey, diSessa, Lehere, \& Schauble, 2003). It seeks to solve problems identified from practice - the difficulties in learning rational numbers and the lack of attention to mental computation in this number set. It is based on a teaching experiment with mental computation 
tasks that provide opportunities for discussing students' strategies and it was carried out in three phases (Figure 2): preparation; experimentation and analysis.

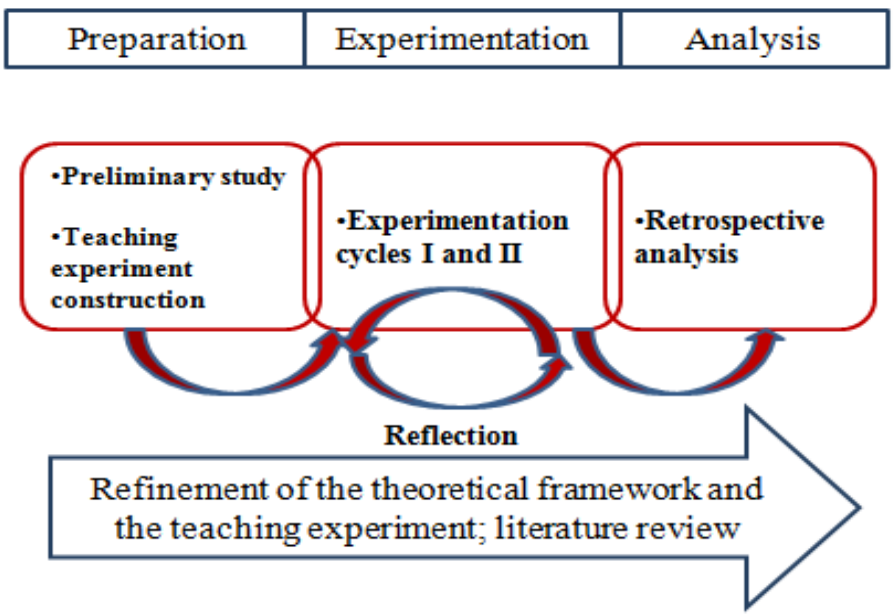

Figure 2. Research design.

In the preparation phase (2010) we undertook a preliminary study in grade 5 to figure out aspects of students' mental computation practice potentially important for planning the teaching experiment. In the second phase (2012-13), two experimental cycles were carried out involving two teachers and two grade 6 classes (39 students) from two different schools, with the first author as a participant observer. During this phase, some refinements were made in the teaching experiment as well as in the conjecture that guides the study. Data was collected using video and audio recordings of the classroom work with mental computation and researcher's notes. Dialogues from audio and 22 video records that show students' strategies were transcribed. We analyzed students' explanations during the collective discussions and coded their mental computation strategies in three main categories shown in Figure 1 (numerical relationships, number facts, and memorized rules) and their mental representations also in three categories (mental models, images, and propositional representations).

The teaching experiment begun with the conjecture that a systematic work with mental computation tasks in mathematics and non-mathematics contexts, involving non-negative rational numbers and whole class discussions in grade 6 helps students to: (i) develop a flexible repertoire of mental computation strategies; (ii) improve their mental computations skills; and (iii) decrease the number of errors that they make in computing mentally. With the two experimental cycles this conjecture evolved to another one stating that grade 6 students' develop mental computation strategies with nonnegative rational numbers when: (i) tasks involve different contexts, representations, and levels of cognitive demand; and (ii) collective discussions about students' strategies are developed to discuss students' errors and to 
construct a set of numerical relationships that may contribute to increase students' repertoire of mental computation strategies that lead them to make less errors.

In the teaching experiment ten mental computation tasks with rational numbers (including number sentences, open number sentences, and/or word problems) were carried out weekly, all prepared by the first author and discussed in detail with the participant teachers. These tasks were presented at the beginning of a mathematics class, using a timed PowerPoint. The students had 15 seconds to solve each number sentence and 20 seconds to solve each word problem individually, in both cases, recording the results on paper (table 1 presents some examples of the tasks used).

Each task has two parts, each one with 5 number sentences or 4 word problems. Upon finishing each part, there was a collective discussion of students' strategies and errors. These mental computation lessons lasted between 30 and 90 minutes.

The teaching experiment was integrated in the lesson plan that each teacher made for his/her class. Therefore, the rational number representation was connected to the mathematical topic that the students were learning at the moment. The tasks that we used begun with the fraction representation (students were working with algebra), then used decimals (students were working with measurement - volume, area, perimeter), and then the percent representation (students were working with statistics). Some of these tasks mixed several rational number representations.

\section{Table 1}

\section{Some Examples of Tasks}

\begin{tabular}{ll}
\hline Think fast! What is the exact value? \\
\hline Task 1: & $1 / 2-?=1 / 4$ \\
\hline Task 3: & $3 / 4+0.5$ \\
\hline Task 6: & $\begin{array}{l}\text { In a cubic box, a face has a perimeter with } 8.8 \mathrm{~cm} . \text { What is the } \\
\text { measure of the side of this face? }\end{array}$ \\
\hline Task 7: & $\begin{array}{l}5 \% \text { of } ?=3 \\
\text { Task 10: }\end{array}$ \\
\hline
\end{tabular}

\section{Students' Strategies and Mental Representations}

Next, we present several situations from the large study that illustrate some students' mental computation strategies with rational numbers as well as the mental representations underlying such strategies, inferred from students' explanations during the collective discussion. Table 2 summarizes the strategies and mental representations that we discuss in detail. 
In the first task, the students were challenged to compute with fractions. To compute " $1 / 2-?=1 / 4$ ", Rui's strategy shows that he used numerical relationships, specifically the change of representation (fraction $\rightarrow$ decimal) and a property of subtraction:" $1 / 2$ is equivalent to 50 cents and $1 / 4$ to 25 cents. It is 25 cents". His strategy suggests that he used a mental model (context of money), which is why he changed fractions to decimals. He also used a propositional representation making a change of representation and subtracting the numbers given to get the subtractive: if $1 / 2=0.5$ and $1 / 4=$ 0.25 so, $1 / 2-?=1 / 4$ is equivalent to $0.5-?=0.25$. Then, $?=0.5-0.25$, although the subtraction is not explicit in his explanation.

\section{Table 2}

Students' Strategies and Mental Representations

\begin{tabular}{|c|c|c|}
\hline Task & Mental computation strategy & $\begin{array}{c}\text { Mental } \\
\text { representation }\end{array}$ \\
\hline \multirow{2}{*}{$1 / 2-?=1 / 4$} & $\begin{array}{l}\text { Rui: Numerical relationships } \\
\text { (change of representation- } \\
\text { fraction } \rightarrow \text { decimal, and a property } \\
\text { of subtraction) }\end{array}$ & $\begin{array}{l}\text { Mental model } \\
\text { (money context) } \\
\text { Propositional } \\
\text { representation }\end{array}$ \\
\hline & $\begin{array}{l}\text { Rogério: Numerical relationship } \\
\text { (relation between numerical } \\
\text { expressions) }\end{array}$ & $\begin{array}{l}\text { Mental image } \\
\text { Propositional } \\
\text { representation }\end{array}$ \\
\hline $3 / 4-1 / 2$ & $\begin{array}{l}\text { João: Numerical relationships } \\
\text { (part-whole relationship and } \\
\text { repeated subtraction) }\end{array}$ & Mental image \\
\hline $\begin{array}{l}\text { In a cubic box, a face } \\
\text { has a perimeter with } \\
8.8 \mathrm{~cm} \text {. What is the } \\
\text { measure of the side of } \\
\text { this face? }\end{array}$ & $\begin{array}{l}\text { Bernardo: Numerical facts (time } \\
\text { table) }\end{array}$ & $\begin{array}{l}\text { Mental model } \\
\text { (money context) }\end{array}$ \\
\hline $5 \%$ of $?=3$ & $\begin{array}{l}\text { João: Numerical relationships } \\
\text { (part-whole relationships) and } \\
\text { numerical facts (time tables) }\end{array}$ & $\begin{array}{l}\text { Mental image } \\
\text { Propositional } \\
\text { representation }\end{array}$ \\
\hline $3 / 4+0.5$ & $\begin{array}{l}\text { Maria: Numerical relationships } \\
\text { (change of representation - } \\
\text { decimal } \rightarrow \text { fraction) }\end{array}$ & $\begin{array}{l}\text { Mental model } \\
\text { (a clock) }\end{array}$ \\
\hline $\begin{array}{l}\text { Ana wants to fill cups } \\
\text { with soda. Each cup has } \\
1 / 8 \text { l of capacity. How } \\
\text { many cups can she fill } \\
\text { with } 0.75 \text { l of soda? }\end{array}$ & $\begin{array}{l}\text { Ricardo: } \text { Numerical } \\
\text { relationships (change of } \\
\text { representation -decimal } \rightarrow \text { percent } \\
\rightarrow \text { fraction) }\end{array}$ & $\begin{array}{l}\text { Propositional } \\
\text { representation }\end{array}$ \\
\hline
\end{tabular}


To compute the same expression, Rogério also used a numerical relationship (relation between numerical expressions), but a mental image, given the details of his explanation: "We have a half of a cake. We ate a half and we keep a quarter. So, it is a half minus a quarter". If we close our eyes and listen to Rogério's explanation, we can imagine in our mind the entire situation described in a concrete way, given the details used by the student "we have a half of a cake" $(1 / 2)$ and "we ate a half" $(1 / 2 \times 1 / 2)$. His strategy also suggests the use of a propositional representation: "if $(1 / 2-(1 / 2 \times 1 / 2))$ $=1 / 4$, so $1 / 2-1 / 4=1 / 4$ ", that expresses some symbolization of the situation (halves and quarters of a cake) and the relation between two numerical expressions, one created by the student $(1 / 2-(1 / 2 \times 1 / 2))$ and the other presented in the task $(1 / 2-?=1 / 4)$.

In the first task, students were challenged to compute with open number sentences, like the one we discussed above, and number sentences like " $3 / 4-1 / 2$ ". To compute this number sentence, João initially used a very common strategy (change $1 / 2$ to $2 / 4$ and then subtract the numerators). But when João was challenged by the teacher to indicate another strategy, he used numerical relationships (part-whole relationship and repeated subtraction) guided by a mental image of a pizza with several pieces removed: "I imagined a pizza in my head, where a piece was missing. A quarter. A quarter was out . . . We removed a half. [We keep] a quarter". João imagined a pizza (the whole) and repeatedly removed pieces of $1 / 4$ and $1 / 2$ of it. He begun with a pizza in his mind, then he removed $1 / 4$ from the whole to represent the $3 / 4$ given in the task and then he removed $1 / 2$ like it is presented in the number sentence.

The context of money seems to be very strong for some students in this study, especially to students from the cycle of experimentation II. For example, task 6 (tables 1 and 2), a word problem was framed in the context of perimeter, but Bernardo ignored this context and used a mental model based on a money context (like Rui did above): "I used money, but I basically did like Aida. I have 4 friends and I need to lend some money to them and so I shared 8.8 and it gives 2.2 [to each of them]". Bernardo wanted to share money, so he divided 8.8 by 4 , probably using numerical facts (time table of 4). However, his explanation is not clear about the way he made the division. In mental computation with percent, students used part-whole relationships (among others) especially in open number sentences. For example, João said: "5\% of ? = 3": "So, I put 3 marbles in a bag. This is 5 [\%]. Then I need, 95 bags more filled with 3 marbles each. No, 20 bags filled with 3 marbles each because 5 times 20 marbles gives $100 \%$. And then I counted the marbles. I did 3 times 20". João's strategy seems to have underlying a mental image based on his daily life experience, as he highlights before presenting it: "With fractions we use a pizza and I remembered one thing from my daily life. I remembered the marbles", which uses a discrete unit (marble bags). This mental image has some specificity: ("3 marbles in a bag", "20 bags filled with 
3 marbles each", "did 3 times 20") allowing us to "imagine" the situation along his explanation. João relates part-whole showing that he knows (according to his mental image) that 5\% corresponds to 3 marbles, and so 20 bags are required (" 20 bags filled with 3 marbles each because 5 times 20 marbles gives 100\%"). Finally, he used numerical facts (time tables) to reach the missing value. This strategy seem to be based also on a propositional representation that reflects the numerical relationships made by João: if 5\% of $?=3$ and $5 \% \times 20=100 \%$ then $3 \times 20=$ ?

One of the most common strategies used by students in tasks involving fractions and decimals (such as $3 / 4+0.5$ ), is the change of representation. While some students prefer to change a fraction to a decimal, others prefer the change a decimal to a fraction. This is the case of Maria:" So, 3/4... I thought in a clock and put 0.50 in half an hour. Then $3 / 4$ is equal to $3 / 4$ of an hour, plus half of an hour gives an hour and a quarter. An hour and a quarter, I put 1.25." Maria's strategy seems to be supported by a mental model (a clock). The sentence "I put 0.50 in half an hour" seems to indicate that she converted the decimal 0.5 in to the fraction $1 / 2$, without performing equivalent fractions or any algorithmic procedure. She solved the operation based on her perception and "mental visualization" of the place of hours on a clock. Further, she indicated the result using decimal (symbolic) and mixed number representation (in natural language) ("An hour and a quarter, I put 1.25"). This strategy shows some skills in the conversion between equivalent representations of rational numbers (decimal $\rightarrow$ fraction $\rightarrow$ decimal) and the use of known contexts to support a mental computation.

The word problem presented in task 10 (tables 1 and 2) could be solved with the expression $0.75 \div 1 / 8$, but Ricardo uses numerical relationships instead the "invert-and-multiply" rule like some of his colleagues did:" $1 / 8$ is a cup and $75 \%$ is $6 / 8 \ldots 6 / 8$ it gives 6 cups". $\mathrm{He}$ changed the decimal representation 0.75 to percent $(75 \%)$ and then to a fraction with an 8 in the denominator $(6 / 8)$ to better compare quantities. Ricardo reaches the result very quickly, showing knowledge about the equivalence of rational number representations. This relationship between rational number representations (fractions, decimal and percent) was discussed several times during the teaching experiment and one of our aims was its development in students. This strategy seems to be based on a propositional representation focused in the change of representation and the relationship between the dividend and the divisor of the division: if $0.75=$ $75 \%=6 / 8$ so, $0.75 \div 1 / 8=6 / 8 \div 1 / 8.6 / 8=6 \times 1 / 8$ so, $6 / 8 \div$ $1 / 8=6$.

\section{Discussion and Conclusion}

We do not know previous studies on students' mental representations underlying their mental computation strategies. Thus, the present study adds 
to the field knowledge about such mental representations and their relation with significant learning experiences, providing important elements to inform curriculum development and teaching practice. In mental computation with rational numbers, when using percent students used mostly strategies based on numerical relationships, such as part-whole relationships, and, when using fractions and decimals, they used change of representation (Caney \& Watson, 2003) and relations between numerical expressions and properties of operations. The analysis of students' strategies shows contexts that are significant for them (e.g., money, clock, marbles, and pizza) and may promote mental representations. Otherwise, such contexts would not be recovered and used in students' strategies. These data (with further examples given in Carvalho, 2016) show that underlying students' strategies we can identify depictive representations such as models or images and descriptive representations such as propositional representations (Schnotz et al., 2010). Mental models arise as a representation of the real world (for example, contexts of clocks and money) without associated specificity. Sometimes, these mental representations are very strong in students' mind, as with Bernardo, who used the context of money even for a problem given in a context of perimeter. Mental images have associated a high specificity and define more precisely the object that they are meant to represent (as marbles and pizza with João and the cake with Rogério). Mental models and images seem to underlie strategies involving numerical relationships like we showed in the case of Rui, Rogério, and João. Propositional representations, as descriptive representations allow us to understand relationships and so they are in the basis of numerical relationships strategies.

Students' explanations suggest several conclusions about their reasoning processes. First, they indicate the power that some mental representations have in the mind of students, supporting their way of reasoning (quite apparent in all students and particularly with Bernardo). Second, the complementarity between depictive and descriptive (Schnotz \& Bannert, 2003; Schnotz et al., 2010) representations seems to be reflected in students' strategies. In João's strategy when he used a mental image (bag of marbles) to establish a relationship based on a propositional representation. In the strategy of Rui, when he used a mental model (context money) to use a property of subtraction based on a propositional representation, and in Rogério's strategy when he used a mental image (a cake) to put into practice a repeated subtraction based on propositional representations. Third, mental representations support students in connecting symbolic representations with contexts, as João who uses a pizza in questions involving fractions and marbles in questions involving percent. Maybe these mental representations were constructed based on an extended work with continuous units in fraction representations, were discrete units were not much used. This stresses the importance to work with both kinds of units, continuous and discrete, in 
several contexts (Bell, 1993), in order to promote a diversity of mental representations in students' minds.

Another thing we want to underline is the importance of the development of numerical relationships in the classroom, especially relationships between rational number representations. To solve a given word problem, Ricardo used a fraction instead of a decimal maybe because the relationships between rational number representations were deeply discussed during the teaching experiment. The development of numerical relationships seemed to support the development of students' propositional representations since the data shows that they are strongly connected.

Concerning the conjecture that guided the teaching experiment, the results show that our ideas evolved in the right direction, although some refinements can be made. To develop mental computation strategies, the students need to work in several contexts (mathematics and non-mathematics), as the results show that a diversity of contexts is important for them according to their mental representations. They also need to work with different rational number representations, and especially in tasks that provide the opportunity to relate them, to increase the flexibility in working with rational numbers. And, finally, they need to work with tasks with different levels of cognitive demand (number sentences, open number sentences, and word problems). One of the refinements to this conjecture may be the addition of continuous and discrete units in students' work as an important aspect to take into account in mental computation tasks (according to the strategies of João). Collective discussions where students' strategies are discussed and developed proved to be important in the process of constructing a set of numerical relationships (as with Ricardo). Those discussions may contribute to increase students' repertoire of mental computation strategies that lead them to make fewer errors.

In this study, students' mental representations reflected important aspects of their knowledge about rational numbers, their operations and relationships, with contexts playing an important role. Mathematical contexts and daily life contexts that students bring to school seem to support their reasoning as we have showed, promoting the development of mental models, images and propositional representations which students use in mental computation.

\section{Acknowledgement}

This study is supported by national funds through a grant provided by to the first author by FCT - Fundação para a Ciência e a Tecnologia (reference FRH/BD/ 9413/2010).

\section{References}

Baddeley, A. (1993). Human memory: Theory and practice. London, UK: Erlbaum. 
Barnett-Clarke, C., Fisher, W., Marks, R., \& Ross, S. (2010). Developing essential understanding of rational numbers: Grades 3-5. Reston, VA: NCTM.

Bell, A. (1993). Principles for the design of teaching. Educational Studies in Mathematics, 24(1), 5-34.

Caney, A., \& Watson, J. M. (2003). Mental computation strategies for partwhole numbers. AARE 2003 Conference papers, International Education Research.

Carvalho, R. (2016). Cálculo mental com números racionais: Um estudo com alunos do 6. ${ }^{\circ}$ ano de escolaridade (Doctoral dissertation, Universidade de Lisboa). Lisboa, Portugal.

Cobb, P., Confrey, J., diSessa, A., Lehere, R., \& Schauble, L. (2003). Design experiments in education research. Educational Researcher, 32(1), 913.

Dehaene, S. (1997). The number sense: How the mind creates mathematics. New York, NY: Oxford University Press.

Empson, S., Levi, L., \& Carpenter, T. (2010). The algebraic nature of fraction: Developing relational thinking in elementary school. In J. Cai \& E. Knuth (Eds.), Early algebraization: A global dialogue from multiple perspectives (pp. 409-428). Heidelberg, Germany: Springer.

Heirdsfield, A. (2011). Teaching mental computation strategies in early mathematics. Young Children, 66(2), 96-102.

Johnson-Laird, P. N. (1990). Mental models. Cambridge, UK: Cambridge University Press.

Plasencia, I. C. (2002). Imágenes mentales en la actividad matemática: Reflexiones de una investigacion. Revista de Didáctica de las Matemáticas, 49, 3-34.

Prediger, S. (2008). The relevance of didactic categories for analyzing obstacles in conceptual change: Revisiting the case of multiplication of fractions. Learning and instruction, 18(1), 3-17.

Schnotz, W., \& Bannert, M. (2003). Construction and interference in learning from multiple representation. Learning and Instruction 13, 141-156.

Schnotz, W., Baadte, C., Müller, A., \& Rasch, R. (2010). Creative thinking and problem solving with depictive and descriptive representations. In L. Verschaffel, E. de Corte, T. Jong, \& J. Elen (Eds.), Use of representation in reasoning and problem solving (pp. 11-35). New York, NY: Routledge.

\section{Authors:}

Renata Carvalho

Universidade de Lisboa

Email: renatacarvalho@campus.ul.pt
João Pedro da Ponte

Universidade de Lisboa

Email:jpponte@ie.ulisboa.pt 